\title{
Spectral behavior of solar oscillations modulated by magnetic variation
}

\begin{abstract}
Heon-Young Chang ${ }^{\star}$
Korea Institute for Advanced Study, 207-43 Cheongryangri-dong Dongdaemun-gu, Seoul 130-012, Korea

Received 12 April 2002 / Accepted 16 May 2002

Abstract. The principal aim of observational helioseismology is to determine mode parameters of the solar oscillations as accurately as possible. Yet estimates of the mode parameters are subject to many sources of noise, including inevitable gaps in data, stochastic nature of excitation processes, interferences among modes. Another uncertainty in frequency determination results from a modulation of the oscillation frequency in itself. It is well known that variation in the mean strength of the solar magnetic field modulates the frequency. We consider effects of the solar magnetic field variation on the spectral behavior of the power spectrum using a simple model. We show that the solar magnetic field variation can cause unwanted sidelobes beside the main peak in the power spectrum, and consequently show that the effect of the frequency modulation due to the solar magnetic field variation can lead to a bias in frequency determinations. This effect should be considered seriously particularly when the $l \neq 0 p$-mode multiplets are analyzed to measure the rotational splitting since the separations in both cases could be comparable. In addition to a bias in frequency estimates the line width is also likely to be overestimated due to a line broadening, which is a measure of the life time of the mode. We therefore suggest that determination of the mode parameters should be derived with due care considering the magnetic field variation when a long data set is analyzed. The frequency modulation should be taken into account in the analysis to make a solid conclusion on any subtle dependence of the mode parameters, such as, that on the solar cycle. We emphasize, however, that a realistic model of the magnetic activity variation over the entire surface of the Sun should be involved in order to predict the effect of the solar magnetic field variation on the spectral profiles of the solar oscillations at the quantitative level. Finally, we conclude by pointing out that a new method is required to accommodate the stochastic force and the phase variation.
\end{abstract}

Key words. methods: data analysis - Sun: activity - Sun: helioseismology - Sun: oscillations

\section{Introduction}

Helioseismology is a study of the solar oscillations sounding the internal structure of the Sun (e.g. Deubner \& Gough 1984). Frequencies of the solar $p$-modes provide the most fundamental and important information on the Sun in that one may infer the solar internal structure and rotation from the observed frequencies (Gough 1984; Christensen-Dalsgaard et al. 1985). To obtain accurate frequencies, it is conventional to take a Fourier power spectrum of time series data obtained from velocity/luminosity variation observations and to measure the frequencies of the peaks in the spectrum by fitting a symmetric Lorentz profile to the observed power spectrum, assuming that those frequencies are constant over the time interval encompassed by the spectrum. This method also yields estimates of the line width and amplitude of the solar oscillations, which include clues of their damping and excitation mechanisms.

The observed power spectrum of the solar oscillations is, however, far from an ensemble of smooth curves. Gaps in the time series, such as, due to the diurnal rotation of the Earth, produce deleterious sidelobes in the power spectrum. The noisy power spectrum requires elaborate data reduction techniques to compensate to some extent for effects of gaps in the data (Brown \& Christensen-Dalsgaard 1990; Lazrek \& Hill 1993; Chang \& Gough 1995; Fossat et al. 1999; Fierry Fraillon \& Appourchaux 2001). Even if continuous data sets without gaps exist, there is still an uncertainty in determining mode parameters due to the stochastic nature of the excitation process of the solar oscillations (Goldreich \& Keeley 1977; Goldreich \& Kumar 1988). Woodard (1984) showed empirically that for the case of a harmonic oscillator excited by random noise the power spectrum will be distributed as the $\chi^{2}$ distribution with two degrees of freedom (for rigorous discussion see Gabriel 1994). That is, the standard deviation of the power at a certain frequency is equal to the mean power at that frequency. What it implies is that even if the length of an uninterrupted data set becomes extended the observed power spectrum may not converge to a smooth curve. Instead the observed power spectrum is likely to be more spiky while the resolution is improved. Alternatively, one may attempt to measure the mode parameters by dividing the whole data set into several shorter data sets and averaging obtained spectra before the fit, since the statistics may become more nearly Gaussian (e.g. Sorensen 1988).

\footnotetext{
* e-mail: hyc@ns.kias.re.kr
} 
Unfortunately, however, this could not be a solution in analyzing the real solar power spectrum. The solar structure undergoes a slow change, causing variation in the frequency. The average of power spectra is simply an average of spectra at different epochs with different frequencies. Therefore, strictly speaking, the obtained frequency from the averaged power spectrum is an average of varied frequencies, and the measured line width is likely to be over-estimated due to such a frequency variation. Woodard \& Noyes (1985) first reported a significant decrease in frequency using solar intensity data from ACRIM (Active Cavity Radiometer Irradiance Monitor), which were obtained from 1980 (near solar maximum) to 1984 (near solar minimum). Since then the correlation between the frequency shift and the solar cycle is firmly established (Libbrecht \& Woodard 1990; Woodard et al. 1991; Bachmann \& Brown 1993; Elsworth et al. 1994; Régulo et al. 1994; Chaplin et al. 1998; Bhatnagar et al. 1999; Howe et al. 1999; Jain et al. 1900; Chaplin et al. 2001). Careful interpretation of frequency dependence on the variation conclusively suggests that the frequency variation is mainly due to the perturbation of the sound speed near the solar surface (Goldreich et al. 1991; Balmforth et al. 1996; Dziembowski et al. 2001).

In this paper, we demonstrate that the variation of the solar magnetic field strength which leads to the frequency modulations of the solar acoustic modes may cause extra noisy peaks in the observed power spectrum in addition to the effects of stochastic excitation and a line to broaden. A general introduction of the frequency modulation can be found in radio communication engineering (e.g. Panter 1965). In helioseismology context the solar magnetic field variation and $g$-modes were considered as an agent of the frequency modulation (Kennedy et al. 1993; Chang 1996; Lou 2001). Previous studies have been concentrated on effects due to the existence of $g$-modes or issues on a time series analysis. We here consider effects of the solar magnetic field variation on the spectral behavior of the power spectrum using a model and also show that the effect of the frequency modulation due to the solar magnetic field variation depends on the solar cycle, as expected in the observed correlation between the frequency shift and the solar cycle. The paper begins with a description of the frequency modulation and presents results of our simulations in Sect. 2. We discuss the effects of the solar magnetic variation on the observed spectrum and conclude in Sect. 3.

\section{Frequency-modulated solar acoustic modes}

As Lou (2001) adopted, one may derive the solar $p$-mode frequency shift due to the solar magnetic modulation in terms of the variational principle formalism (Chandrasekhar 1964). The instantaneous angular frequency $\omega(t)$ of a particular $p$-mode modulated by the solar magnetic field variation can be given as $\omega(t)=\omega_{0}+\epsilon f(t)$, where $\omega_{0}$ is the unmodulated angular frequency ${ }^{1}, \epsilon$ is the strength of the deviation which is assumed to be related to mode properties, such as, inertia, and $f(t)$ is a function which is assumed to vary with the solar magnetic field strength. We note that $\epsilon$ is so small that the modulus of $\omega_{0}$ is

\footnotetext{
${ }^{1}$ an angular frequency $\omega_{0}$ is $2 \pi$ times a cyclic frequency $v_{0}$.
}

much greater than that of $\epsilon f(t)$, and $f(t)$ is a slowly varying function compared to the $p$-mode and same for all the solar $p$-modes since all the solar oscillations contributing to the signal are experiencing the same perturbation agent to the acoustic cavity. We used solar magnetic field data ${ }^{2}$ available at the National Solar Observatory at Kitt Peak to generate $f(t)$, converting the observed magnetic field strength to the frequency variation with the linear relation obtained by Woodard et al. (1991). Though the apparent periodicity is most likely related to presence of active regions that corotate with the Sun, we presume that this feature simulate intrinsic periodic variations of solar magnetic field strengths that may lead to frequency modulations of solar $p$-modes. It should be pointed out, however, that one should employ a more detailed model for the distribution of magnetic field in the solar interior to estimate actual biases of frequency and line width determinations. The relation between the magnetic field strength and the frequency shift may not be an optimal function (Bhatnagar et al. 1999), but any subtle discrepancy should not be a serious problem in this demonstration. Since Woodard et al (1991) normalized the relation to $l=0$ mode of $\approx 3 \mathrm{mHz}$, in our simulation $\epsilon$ may vary a factor of two depending on $\omega_{0}$ we take. The strength of the deviation $\epsilon$ is roughly an increasing function with the frequency. The simulated signal of the frequency-modulated $p$-mode can be written as

$I_{\mathrm{FM}}(t)=A(t) \cos \left(\omega_{0} t+\epsilon \int_{0}^{t} f(\tau) \mathrm{d} \tau+\psi_{0}\right)$,

where $A(t)$ is an amplitude which we consider to be determined such that the energy distribution follows the Boltzmann distribution, and $\psi_{0}$ is a phase when $t=0$. Without loss of generality the phase constant can arbitrarily be set equal to zero and $A(t)$ constant $A_{0}$.

Since an arbitrary continuous function $f(t)$ can be expressed in terms of harmonics, according to Fourier's theorem, we begin with a single-tone sinusoid as an illustration, such that $f(t)=\cos \omega_{\mathrm{m}} t$. Then the frequency-modulated function is given by

$I_{\mathrm{FM}}(t)=A_{0} \cos \left(\omega_{0} t+\beta \sin \omega_{\mathrm{m}} t\right)$,

where $\beta$ is $\epsilon / \omega_{\mathrm{m}}$ so that the maximum phase deviation is inversely proportional to the frequency of the modulating function. The representation for $I_{\mathrm{FM}}(t)$ can be expanded due to Jacobi's expansion in a series of Bessel coefficients using the Fourier series expansion (Watson 1922). Using a property of Bessel functions we have

$I_{\mathrm{FM}}(t)=A_{0} \sum_{n=-\infty}^{\infty} J_{n}(\beta) \cos \left(\omega_{0} t+n \omega_{\mathrm{m}} t\right)$

where $J_{n}(\beta)$ is the Bessel function of the first kind with $J_{n}(\beta)=$ $(-1)^{n} J_{-n}(\beta)$. Thus we have a time function consisting of a main unmodulated function and an infinite number of sidebands, whose relative amplitudes are proportional to $J_{n}(\beta)$ spaced at frequencies $\pm n \omega_{\mathrm{m}}$. As $\beta \rightarrow \infty$, the number of sidebands increases and the spectral components become more and more confined between $\omega_{0} \pm \epsilon$. And the maximum height is also

\footnotetext{
${ }^{2}$ ftp://argo.tuc.noao.edu/kpvt/daily/stats/mag.dat
} 

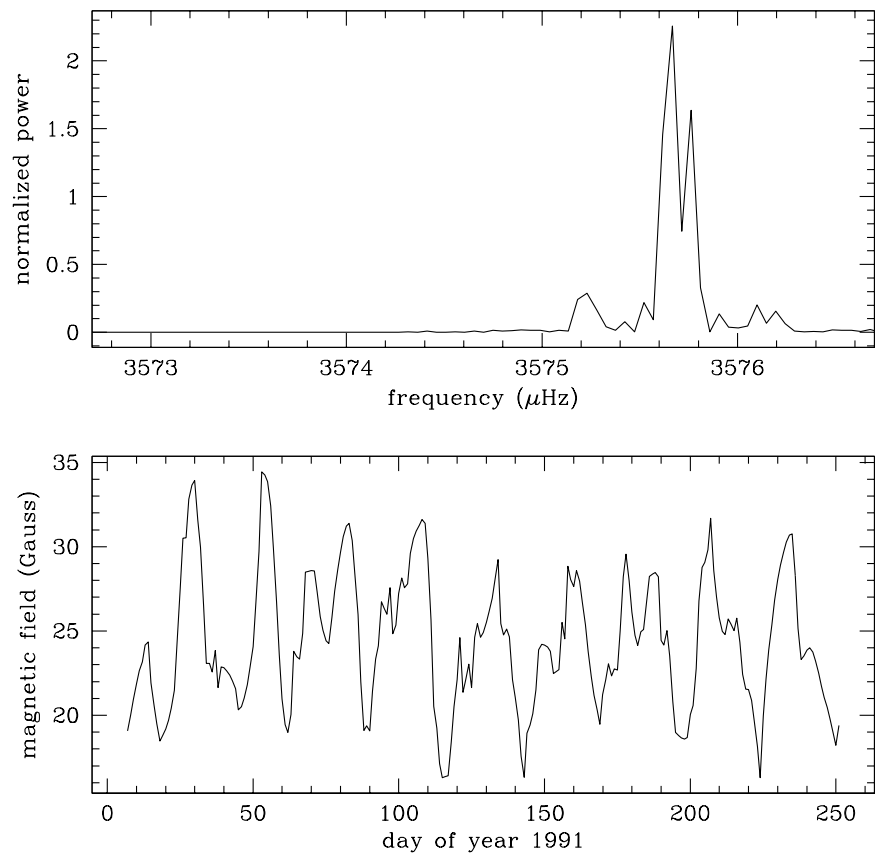

Fig. 1. Simulated power spectrum of the frequency-modulated mode is shown in the upper panel. The spectral line is significantly broadened and apparent sidelobes are spaced at $\sim 0.4 \mu \mathrm{Hz}$. Note that power is arbitrarily normalized and noise-free. The frequency modulation function $f(t)$ is generated from the magnetic field strength observed in 1991 (near solar maximum) which is shown in the lower panel.

reduced because energy tends to be distributed among many small peaks. Note that $\beta$ is proportional to the sensitivity of the mode on the solar magnetic variation. In reality, the modulating function is multitones in nature. The signal suffers more interference, causing more sidebands. Obviously the power spectrum of a modulated function depends on a shape of the modulation function. For instance, in the case where the frequency modulation is a square wave function, when the amplitude of the square wave $\Delta \omega$ is much larger than the fundamental frequency of the square wave the power spectrum shows two distinctive peaks in the vicinity of $\omega_{0} \pm \Delta \omega$.

In Figs. 1 and 2 we show the Fourier power spectra of simulated data generated by Eq. (1). In our simulations we presume $l=0$ mode with its cyclic frequency $3574.7 \mu \mathrm{Hz}$ and set $\epsilon=2$ as the effect of the frequency-modulation should be more serious in higher frequency modes. The duration of the observation corresponds to 8 months. As mentioned earlier, we take the observed magnetic field strength and translate it into $f(t)$ according to the linear relation given by Woodard et al. (1991). We show two specific cases of different epochs, that is, year 1991 (near solar maximum) and 1997 (near solar minimum) to demonstrate its dependence on the solar cycle. The observed magnetic field strengths we adopt have been shown in the lower panels separately in Figs. 1 and 2. Besides the obvious frequency shifts, which can be defined such that $T^{-1} \int_{0}^{T} \omega(t) \mathrm{d} t-\omega_{0}$ where $T$ is the interval of observation, unexpected sidelobes appear (Fig. 1). In the case of year 1991, the solar magnetic modulation is almost a single-tone sinusoid with $\nu_{\mathrm{m}} \approx 0.4 \mu \mathrm{Hz}$, which is a similar value of the amplitude of $\epsilon f(t)$ in Eq. (2). In other words, the peak phase deviation $\beta$
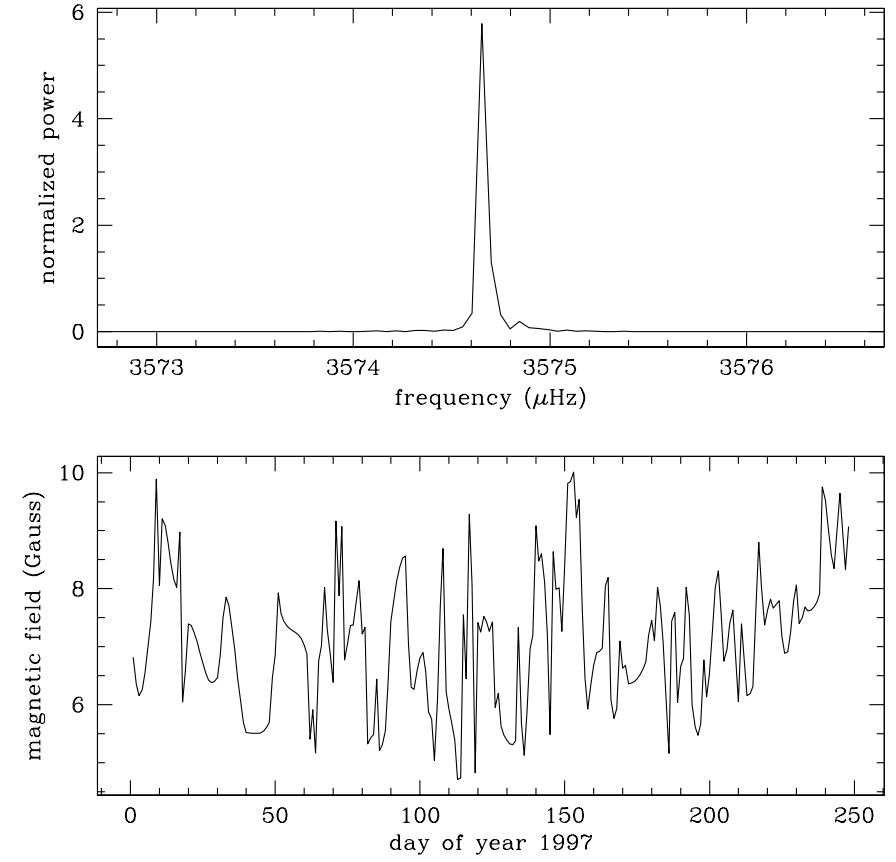

Fig. 2. Similar plots with Fig. 1, except that the frequency modulation function is derived from the data obtained in 1997 (near solar minimum). Sidelobes are less significant than those in Fig. 1, yet the spectral line is still broadened.

is $\sim 1$. This fact results in sidelobes located at $\sim 0.4 \mu \mathrm{Hz}$ apart from the main peak. The effect of the frequency modulation is likely more conspicuous in the case of the solar maximum. It should be noted, as we mentioned above, that these specific sidelobes at $\sim 0.4 \mu \mathrm{Hz}$ may not be manifest in the real solar power spectrum since the magnetic variation used here with periods of $\sim 30$ days results from the apparent periodicity due to the presence of active regions corotating with the Sun. In Fig. 2, the only possibly detectable effect of the frequency modulation is a broadening of the main peak. In this particular case, the spectral line is more broadened by a factor of two than that in the case where there is no frequency-modulation. One important thing to bear in mind is, therefore, that according to the simulated power spectrum the spectral line width is likely to be overestimated in any case if the effect of the frequency modulation is ignored. Moreover, when the mode is frequencymodulated the height of the central peak is also likely reduced since the energy of the mode tends to be distributed among many small sidelobes as shown above. We note that a decrease in the strength of the modes from solar minimum to maximum is reported by the BiSON group, where the effect of the frequency modulation has been ignored in the analysis (Elsworth et al. 1993). The frequency modulation should be taken into account to make a solid conclusion on this matter. Otherwise, estimates of the life time and the strength of the solar oscillations seems likely to be biased.

\section{Discussion and conclusion}

Magnetic activity changes the outer layers of the solar envelope, modifying the resonant properties of the $p$-mode cavity and modulating the oscillation frequencies. By simply 


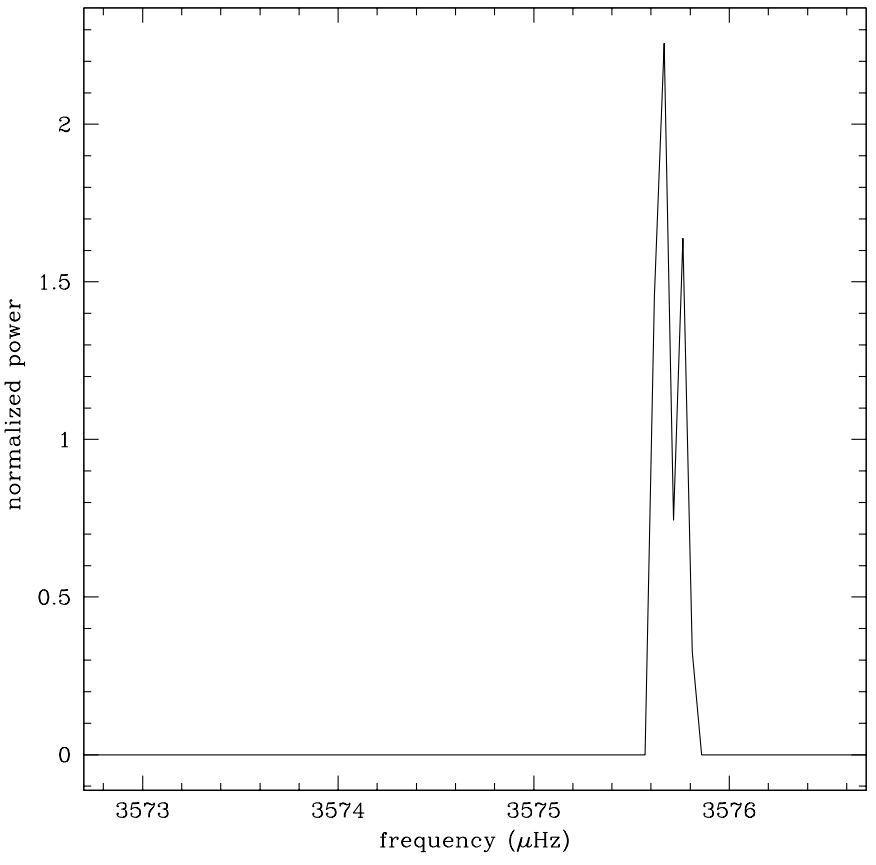

Fig. 3. Similar power spectrum shown in Fig. 1, except that all other noisy structures further than $\pm 0.2 \mu \mathrm{Hz}$ from the main peak are set to zero.

measuring the positions of peaks in power spectra, one is restricted by the uncertainty in frequency determination due to the phase wandering induced by the changing cavity. We demonstrate that the frequency modulation by the solar magnetic field variation may affect estimates of the mode parameters, particularly during a period near the solar maximum. This effect should be seriously taken into consideration when the $l \neq 0 p$-mode multiplets are analyzed to measure the rotational splitting. As shown above the sidelobes due to the solar magnetic variation may be displaced next to the main peak at similar amounts of the rotationally splitting separation if the magnetic field varies with a roughly 30 -day timescale. Although many attempts to correlate the solar rotation curve with the solar cycle have been made (Jiménez et al. 1994; Chaplin et al. 1996; Antia \& Basu 2000), we suggest that conclusions of the dependence of the solar rotation curve on the solar cycle should be derived with due care since the mode estimatation could be biased due to the frequency modulation as well as the mode interference (Appourchaux et al. 2000). In addition to a bias in frequency estimates, the line width is also likely to be overestimated. In Fig. 3, we show the power spectrum shown in Fig. 1, in which all noisy structures further than $\pm 0.2 \mu \mathrm{Hz}$ from the main peak are set to zero, in order to emphasize the line broadening and to suppress the possible rotational effect.

We conclude by pointing out that there was an attempt for developing a method to measure the frequency by fitting the temporal signal to a physical model deduced by the superposition of many modes, rather than using its power spectrum (Chang 1996; Chang \& Gough 1995). This technique uses the fact that the unknown components to the wandering of amplitudes and phases of constituent oscillators arising from the temporal variation of the cavity in which they are confined are related to each other in a known way. Provided that a sophisticated algorithm is implemented to accommodate the stochastic force and the surface variation causing the phase modulation, such an idea may yield more accurate estimates of the frequency and the life time of the solar short period oscillations.

Acknowledgements. We would like to thank Douglas Gough and Takashi Sekii for useful discussions while the idea is conceived, and the anonymous referee for critical comments which improve the original manuscript. NSO/Kitt Peak magnetic data used here are produced cooperatively by NSF/NOAO, NASA/GSFC and NOAA/SEL.

\section{References}

Antia, H. M., \& Basu, S. 2000, ApJ, 541, 442

Appourchaux, T., Chang, H.-Y., Gough, D. O., \& Sekii, T. 2000, MNRAS, 319, 365

Bachmann, K., \& Brown, T. 1993, ApJ, 411, L45

Balmforth, N. J., Gough, D. O., \& Merryfield, W. J. 1996, MNRAS, 278, 437

Bhatnagar, A., Jain, K., \& Tripathy, S. C. 1999, ApJ, 521, 885

Brown, T. M., \& Christensen-Dalsgaard, J. 1990, ApJ, 349, 667

Chandrasekhar, S. 1964, ApJ, 139, 664

Chang, H.-Y. 1996, Ph.D. Thesis, University of Cambridge, Cambridge

Chang, H.-Y., \& Gough, D. 1995, in Proc. Fourth SOHO Workshop Helioseismology, ed. J. T. Hoeksema et al., ESA SP-376 (Noordwijk: ESA), 2, 179

Chaplin, W. J., et al. 1996, MNRAS, 283, L31

Chaplin, W. J., et al. 1998, MNRAS, 300, 1077

Chaplin, W. J., et al. 2001, MNRAS, 324, 910

Christensen-Dalsgaard, J., et al. 1985, Nature, 315, 378

Deubner, F.-L., \& Gough, D. 1984, ARA\&A, 22, 593

Dziembowski, W. A., Goode, P. R., \& Schou, J. 2001, ApJ, 553, 897

Elsworth, Y., et al. 1993, MNRAS, 265, 888

Elsworth, Y., et al. 1994, ApJ, 434, 801

Fierry Fraillon, D., \& Appourchaux, T. 2001, MNRAS, 324, 1159

Fossat, E., et al. 1999, A\&A, 343, 608

Gabriel, M. 1994, A\&A, 287, 685

Goldreich, P., \& Keeley, D. 1977, ApJ, 212, 243

Goldreich, P., \& Kumar, P. 1988, ApJ, 326, 462

Goldreich, P., Murray, N., Willette, G., \& Kumar, P. 1991, ApJ, 370, 752

Gough, D. 1984, Phil. Trans. R. Soc. Lond. A, 313, 27

Howe, R., Komm, R., \& Hill, F. 1999, ApJ, 524, 1084

Jain, K., Tripathy, S. C., \& Bhatnagar, A. 2000, ApJ, 542, 521

Jiménez, A., et al. 1994, ApJ, 435, 874

Kennedy, J. R., Jefferies, S. M., \& Hill, F. 1993, in GONG 1992: Seismic Investigation of the Sun and Stars, ed. T. M. Brown (San Francisco: ASP), ASP Conf. Ser., 42, 273

Lazrek, M., \& Hill, F. 1993, A\&A, 280, 704

Libbrecht, K. G., \& Woodard, M. F. 1990, Nature, 345, 779

Lou, Y.-Q. 2001, ApJ, 56, L121

Panter, P. F. 1965, Modulation, Noise, and Spectral Analysis (New York: McGraw-Hill)

Régulo, C., et al. 1994, ApJ, 434, 384

Sorensen, J. 1988, in Seismology of the Sun and Sun-like Stars, ed. E. J. Rolfe, ESA SP-286 (Noordwijk: ESA), 41

Watson, G. 1922, Theory of Bessel Function (Cambridge: Cambridge Univ. Press)

Woodard, M. 1984, Ph.D. Thesis, University of California, San Diego Woodard, M., \& Noyes, R. 1985, Nature, 318, 449

Woodard, M., Kuhn, J., Murray, N., \& Libbrecht, K. 1991, ApJ, 373, L81 